\title{
Highlights on eclipsing binary variables from Araucaria Project
}

\author{
Paulina Karczmarek ${ }^{1}$, Grzegorz Pietrzyński ${ }^{1,2}$, Wolfgang Gieren ${ }^{2}$, Bogumił Pilecki ${ }^{1}$, Dariusz \\ Graczyk $^{2}$, Ian Thompson ${ }^{3}$, Marek Górski ${ }^{1}$, Piotr Konorski ${ }^{1}$, Ksenia Suchomska ${ }^{1}$, Nicholas Nardetto ${ }^{4}$, \\ and Radoslaw Smolec ${ }^{5, a}$ \\ 1 Warsaw University Observatory, Al. Ujazdowskie 4, 00-478 Warsaw, Poland \\ 2 Departamento de Astronomia, Universidad de Concepción, Casilla 160-C, Concepción, Chile \\ 3 Carnegie Observatories, 813 Santa Barbara Street, Pasadena, CA 91101-1292, USA \\ ${ }^{4}$ Laboratoire Fizeau, UNS/OCA/CNRS UMR6525, Parc Valrose, 06108 Nice Cedex 2, France \\ 5 Copernicus Astronomical Centre, Polish Academy of Sciences, Bartycka 18, 00-716 Warsaw, Poland
}

\begin{abstract}
The Araucaria Project, which main goal is to provide precise determination of the cosmic distance scale, has recently made a set of discoveries involving variable stars in binary systems. Among these discoveries we highlight three: $1 \%$ precise measurement of a Cepheid's dynamical mass and its projection factor, accurate determination of both stellar and orbital parameters of eclipsing binary consisting of two Cepheid variables, and discovery of new class of variable stars, mimicking RR Lyrae pulsators.
\end{abstract}

Key words. stars: Cepheids - stars: pulsation - stars: eclipsing binaries - stars: distances - stars: oscillations

\section{The Araucaria Project and the recent results}

The Araucaria project is a long-term observational program which aims to provide an improved local calibration of the extragalactic distance scale. An application of a number of different stellar standard candles (Cepheid variables, RR Lyrae stars, red clump giants, and blue supergiants) allows to independently determine distances to nearby galaxies. Furthermore, the distances obtained with different methods i.e. using different stellar candles, are compared to trace down the dependencies on environmental properties of the various standard candles. Standard candles in eclipsing binary systems are of particular interest, as they provide both orbital and stellar parameters of high accuracy, which may have an impact upon the theory of stellar pulsation and evolution. The following is the summary of selected results recently obtained by the Araucaria project, specifically focused on pulsating variables in binary systems.

The two ways to determine the Cepheid mass - from stellar pulsation theory and from stellar evolution theory - yield the results different by $30 \%$ (the Cepheid mass discrepancy problem [1]). The accuracy of previous efforts to establish a dynamical Cepheid mass from single-lined non-eclipsing binaries was typically about $15-30 \%$, which was not good enough to resolve the mass discrepancy problem [2]. The discovery of an eclipsing binary system OGLE-LMC-CEP0227 [3] consisting of a classical Cepheid pulsating with a period of 3.8 days together with a stable red giant in a 310-day orbit allowed to determine the mass of the pulsator to $4.165 \mathrm{M}_{\odot}$ with a precision of $1 \%$ [4]. This determination agrees with its pulsation mass, providing strong evidence that pulsation theory correctly and precisely predicts the masses of classical Cepheids. For the first time a direct, geometrical and distance-independent determination of the Cepheid projection factor needed for Baade-Wesselinktype analyses was possible. The $p$-factor value $p=1.21 \pm 0.03$ (stat.) \pm 0.04 (syst.) is consistent with theoretical expectations for a short period Cepheid and interferometric measurements for $\delta$ Cep [5].

\footnotetext{
a e-mail: smolec@camk.edu.pl
} 

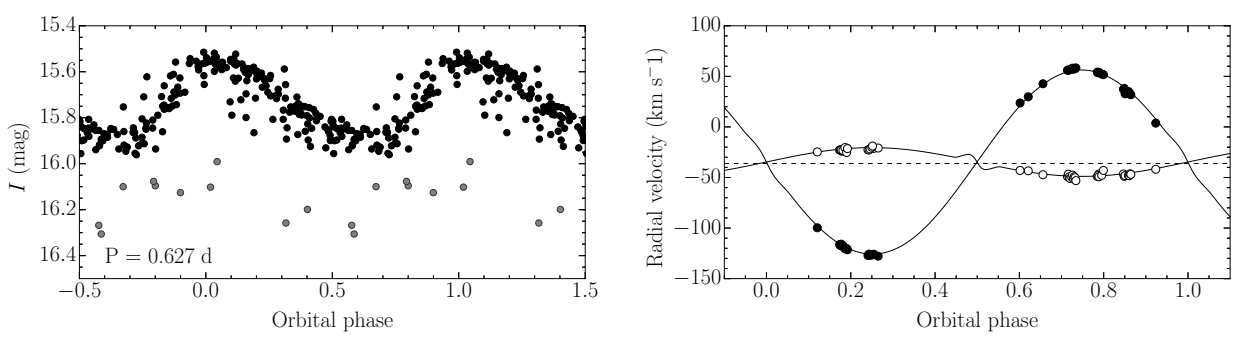

Figure 1. Left: Pulsational $I$-band light curve of the primary component, folded with a pulsation period of 0.627 days. Gray points correspond to eclipses. Right: Disentangled orbital radial velocity curves of both components of BEP system. Filled and open circles denote primary and secondary component, respectively. Solid line shows a least square fit to the measured velocities.

Recent discovery of the first eclipsing binary consisting of two classical Cepheid variables OGLE-LMC-CEP-1718 [3] is an important step towards better understanding of binary pulsators. A detached system of 413-day eccentric $(e=0.276)$ orbit consists of two overtone pulsators, with pulsation periods of 1.96 and 2.48 days. The orbital solution yields dynamical masses of $3.30 \pm 0.11 \mathrm{M}_{\odot}$ and $3.28 \pm 0.11 \mathrm{M}_{\odot}$ respectively, which are equal to within 1.5\% [6]. The existence of two equal-mass Cepheids in a binary system having different pulsation periods poses an interesting challenge to stellar evolution and pulsation theories. Ongoing detailed study of this system, using additional data sets, should yield deeper insight about the physics of stellar evolution of Cepheid variables.

Binary Evolution Pulsator (BEP) refers to a detached binary system which, after the episode of mass transfer, consists of a low-mass pulsating component and its companion. The first of its kind, OGLE-BLG-RRLYR-02792 [7], discovered in 2012 in the Galactic Bulge, exhibits RR Lyrae-like oscillations (Fig. 1) while having a mass of only $0.26 \mathrm{M}_{\odot}$ [8]. As the stellar evolution theory fails to explain RR Lyrae-like pulsations emerging from stars with core mass less than $0.45 \mathrm{M}_{\odot}$, the theory of binary interactions i.e. the mass transfer between companions, may serve an alternative explanation. The system evolved from a close binary with an initial orbital period of 2.9 days, underwent the mass transfer episode from initially more massive primary to a secondary, and as a result, the primary became a low-mass hot helium core with a thin hydrogen envelope. Its temperature and luminosity place it in the same area of the instability strip in the Hertzsprung-Russell diagram as that occupied by RR Lyrae stars. The presence of BEPs may affect the distance measurements with RR Lyrae stars. Our estimations show that $0.2 \%$ of RR Lyrae stars may be recognized as BEPs, therefore distances measured with RR Lyrae stars should be distorted, but a significant change is not expected.

\section{Acknowledgements}

We acknowledge support from the Polish National Science Center grant MAESTRO 2012/06/A/ST9/00269 and the Polish Ministry of Science grant and Higher Education through the program "Ideas Plus" award No. IdP2012 000162.

\section{References}

1. G. Bono, W. Gieren, M. Marconi, et al., ApJ 563 (2001), 319-324

2. R. E. Nancy, AIP Conf. 1170 (2009), 69-72

3. I. Soszyński, R. Poleski, A. Udalski, et al., Acta Astron. 58 (2008), 163-185

4. G. Pietrzyński, I. B. Thompson, W. Gieren et al., Nature 468 (2010), 542-544

5. B. Pilecki, D. Graczyk, G. Pietrzyński et al., MNRAS 436 (2013), 953-967

6. W. Gieren, B. Pilecki, G. Pietrzyński et al., ApJ 786 (2014), 80-87

7. I. Soszyński, W. A. Dziembowski, A. Udalski et al., Acta Astron. 61 (2011), 1-23

8. G. Pietrzyński, I. B. Thompson, W. Gieren et al., Nature 484 (2012), 75-77 